\title{
Survivorship Care Plan
}

National Cancer Institute

\section{Source}

National Cancer Institute. Survivorship Care Plan. NCI Thesaurus. Code C157525.

A document that contains a history of an individual's cancer treatment history as well as guidelines for monitoring and maintaining their health, including the need for future check-ups and cancer tests. The information may also include potential long-term late effects of the treatment received. 